\title{
An Efficient Blind Estimation of Carrier Frequency Offset in OFDM Systems
}

\author{
Said Lmai, Student Member, IEEE, Arnaud Bourré, Christophe Laot, Senior Member, IEEE, \\ and Sebastien Houcke, Member, IEEE,
}

\begin{abstract}
In this paper, we propose a low-complexity blind carrier frequency offset (CFO) estimation scheme, for constant modulus (CM) signaling based orthogonal frequency division multiplexing (OFDM) systems. Provided that the channel can be assumed to be slowly time-varying, subcarriers having the same indices in two consecutive OFDM symbols will experience nearly the same channel effect. This assumption enables us to derive a cost function that is determined by the sum of the products of the signal amplitudes on each pair of equivalent subcarriers from two successive OFDM symbols. The maximization process of this cost function makes it possible to find an appropriate estimate of the CFO. Over frequency-selective Rayleigh fading channels, the proposed CFO estimation method provides improved performance over existing techniques. Moreover, in contexts of narrowband noise and signal gain variations, simulations demonstrate the robustness and immunity of our scheme.
\end{abstract}

\section{INTRODUCTION}

$\mathbf{T}$ He multicarrier modulation technique in the form of orthogonal frequency division multiplexing (OFDM) is an efficient scheme adopted in several standards for systems demanding high data rate. In addition to its simple implementation when using the FFT algorithm for discrete Fourier transform (DFT), the OFDM system is also known for its relatively low sensitivity to time synchronization errors. However, in the presence of carrier frequency offset (CFO), orthogonality between subcarriers is quickly destroyed and inter-carrier interference (ICI) arises, resulting in bit error rate performance loss. CFO is mostly due to transmitter-receiver relative movement, channel variability and the imperfect alignment between local transmitter and receiver oscillators.

Several CFO estimation techniques have been proposed in the literature. They are either data-aided [1]-[4] or simply blind [5]-[10]. This second category, also referred to as nondata-aided, may rely on inserting null subcarriers (e.g. [5] and references therein) and is then power-efficient, or can be bandwidth-efficient in the case where the OFDM system is fully loaded. Additionally, [11]-[15] have proposed estimators operating over post-DFT signal. In [12], the authors assume that the channel response on two adjacent subcarriers remains practically unchanged. Therefore, over each OFDM symbol,

Copyright (c) 2013 IEEE. Personal use of this material is permitted. However, permission to use this material for any other purposes must be obtained from the IEEE by sending a request to pubs-permissions@ieee.org.

S. Lmai, A. Bourre, C. Laot, and S. Houcke are with the Institut Mines-Telecom, Telecom Bretagne, UMR CNRS 6285 Lab-STICC, Technopôle Brest-Iroise - CS 83818, 29238 Brest Cedex 3, France (e-mail: said.lmai@telecom-bretagne.eu).

This work was supported in part by the 'Fonds unique interministériel' (FUI) France within COMET project. they proceed by using the power difference minimization between two neighboring subcarriers to develop an estimator adapted to constant modulus (CM) signaling. The same authors introduced in [13] another estimator that exploits the slow channel changes in time domain over two consecutive OFDM symbols. The differential OFDM system was the context of use discussed in the paper [13]. The same estimator is proposed in [15] with further developments and without the constraint of differential modulation. As reported in [16], let PDE-F (power difference estimator in frequency domain) and PDE$\mathrm{T}$ (its analog in time domain) denote the CFO estimators proposed in [12] and [15], respectively. By minimizing the power difference between all pairs of subcarriers, adjacent subcarriers in each OFDM symbol (PDE-F) or having the same indices in two successive OFDM symbols (PDE-T), estimation functions are derived.

In this paper, we propose a novel, efficient blind CFO estimator for fully loaded CM-OFDM systems. The basic assumption used to derive the cost function is always that the channel taps change slowly in time domain. Provided that the channel response remains almost constant over two successive OFDM symbols, we initially assume the minimization of the difference in post-DFT signal amplitudes between all pairs of subcarriers having the same indices. Analogously to PDE-F and PDE-T, let use ADE-T refer to the proposed estimator while it stands for amplitude difference estimator in time domain. Using the exhaustive search method, we show that PDE-F, PDE-T and ADE-T performance achievements are almost identical. Likewise, the cost function obtained for ADE-T has quasi-regular shape and is simple to implement. It can be closely approximated by a sinusoid with a global maximum corresponding to the desired CFO estimate value. The low-complexity curve-fitting method as introduced by [11] and used in [12] and [15] can be efficiently employed. Compared to PDE-F and PDE-T, ADE-T performance is identified over frequency-selective fading channels. The realized gain indicates the reliability of the estimate while applying an appropriate trade-off between performance and complexity. Moreover, ADE-T is less sensitive to noise and insensitive to gain fluctuations as discussed in the Section III.

The remainder of the paper is structured as follows. Section II is devoted to describing the system model, including the CFO problem formulation. In Section III, we present the proposed CFO estimation technique. Simulation results are given in Section IV. Finally, Section V concludes the paper. Throughout this paper, we denote: $(.)^{T}$ for complex transposition, $(.)^{*}$ for complex conjugation, $(.)^{H}$ for complex conjugate 
transposition, |.| for complex modulus or absolute value (of a real), $\mathbf{I}_{N}$ for $N \times N$ identity matrix, $\operatorname{diag}($.$) for diagonal$ matrix whose entries are the elements of (.), and $:=$ for equal by definition.

\section{System MODEL}

Let us consider a conventional OFDM transmission system with $N$ orthogonal subcarriers. The $l$ th OFDM symbol carries off $N$ information symbols gathered as follows $\mathbf{d}_{l}=\left[d_{l}^{0}, d_{l}^{1}, \ldots, d_{l}^{N-1}\right]^{T}$, after being drawn uniformly from a CM constellation. Then, the modulation of vector $\mathbf{d}_{l}$ is performed and the inverse-DFT (I-DFT) operation output is $\mathbf{u}_{l}=\left[u_{l}^{0}, u_{l}^{1}, \ldots, u_{l}^{N-1}\right]^{T}$. Inserting a $T_{c p}\left(\right.$ resp. $\left.N_{c p}\right)$ duration (resp. number of samples) $\mathrm{CP}$ will follow and the total duration of the obtained OFDM symbol to transmit is $T_{t}=T_{u}+T_{c p}$ (resp. $N_{t}=N+N_{c p}$ ), for a useful part of $T_{u}$ length and a sampling interval of $T_{s}=\frac{T_{u}}{N}$. Thus, the $N$ useful samples of the $l$ th baseband OFDM symbol are:

$$
u_{l}^{k}=\frac{1}{\sqrt{N}} \sum_{n=0}^{N-1} d_{l}^{n} e^{j 2 \pi \frac{n}{N} k}, \quad k=0,1, \ldots, N-1
$$

expression obtained from:

$$
\mathbf{u}_{l}=\mathbf{W d}_{l}, \quad l=1,2, \ldots
$$

where $\mathbf{W}$ is the $N \times N$ normalized I-DFT matrix, whose elements are $W_{m, n}=(1 / \sqrt{N}) \exp (j 2 \pi m n / N)$ by the $m$ th row and the $n$th column along with $m, n=0,1, \ldots, N-1$. Afterwards, the signal so far constructed passes through the multipath fading channel, having the corresponding frequency response $\mathbf{H}_{l}=\operatorname{diag}\left(\left[H_{l}^{0}, H_{l}^{1}, \ldots, H_{l}^{N-1}\right]\right)$. Let $\epsilon$ denote the introduced $\mathrm{CFO}$, which is normalized with respect to the subcarrier spacing (in this case $\frac{1}{T_{u}}$ ). Thus, $\epsilon \in$ $(-0.5,0.5)$ and the resulting accumulated phase shift on the OFDM symbol time domain samples is depicted by $\mathbf{C}(\epsilon)=$ $\operatorname{diag}\left(\left[e^{j 2 \pi \frac{\epsilon}{N} \times 0}, e^{j 2 \pi \frac{\epsilon}{N} \times 1}, \ldots, e^{j 2 \pi \frac{\epsilon}{N} \times(N-1)}\right]\right)$. Removing the $\mathrm{CP}$ is the first operation performed at reception and the retained signal is $\mathbf{y}_{l}=\left[y_{l}^{0}, y_{l}^{1}, \ldots, y_{l}^{N-1}\right]^{T}$. This received OFDM symbol might be described as:

$$
\mathbf{y}_{l}=e^{j 2 \pi \frac{\epsilon}{N}(l-1)\left(N+N_{c p}\right)} \mathbf{C}(\epsilon) \mathbf{W} \mathbf{H}_{l} \mathbf{d}_{l}+\mathbf{v}_{l},
$$

where $\mathbf{v}_{l}=\left[v_{l}^{0}, v_{l}^{1}, \ldots, v_{l}^{N-1}\right]^{T}$ is the vector of white Gaussian noise with zero mean and variance $\sigma_{v}^{2}$. Note that the resulting common phase shift relative to the $l$ th OFDM symbol is given by $e^{j 2 \pi \frac{\epsilon}{N}(l-1)\left(N+N_{c p}\right)}$. In the case of perfect time synchronization, CFO is estimated and then compensated before conducting the DFT operation. In other words, $\epsilon$ is firstly estimated by $\hat{\epsilon}$ and after that, $\mathbf{y}_{l}$ is mutiplied by $\mathbf{C}(\hat{\epsilon})^{*}$ for CFO compensation and $\mathbf{W}^{H}$ for the DFT process. As a result, we get the vector $\mathbf{s}_{l}=\left[s_{l}^{0}, s_{l}^{1}, \ldots, s_{l}^{N-1}\right]^{T}$ defined by:

$$
\mathbf{s}_{l}=\mathbf{W}^{H} \mathbf{C}(\hat{\epsilon})^{*} \mathbf{y}_{l}
$$

and the $k$ th element is:

$$
s_{l}^{k}=\frac{1}{\sqrt{N}} \sum_{m=0}^{N-1} y_{l}^{m} e^{-j 2 \pi \frac{m}{N}(\hat{\epsilon}+k)}, k=0,1, \ldots, N-1
$$

where:

$$
\begin{gathered}
y_{l}^{m}=\frac{e^{j 2 \pi \frac{\epsilon}{N}(l-1)\left(N+N_{c p}\right)}}{\sqrt{N}} \sum_{n=0}^{N-1} d_{l}^{n} H_{l}^{n} e^{j 2 \pi \frac{n+\epsilon}{N} m}+v_{l}^{m}, \\
m=0,1, \ldots, N-1
\end{gathered}
$$

As in [12] and [15], developments hereinafter are made under the noise-free assumption.

\section{Proposed CFO ESTIMATION SCHEME}

At reception, a proper CFO compensation allows us to have an ICI-free signal after the DFT operation. In terms of literary formulation, if $\hat{\epsilon}=\epsilon$ and since $\mathbf{C}(\hat{\epsilon})^{*} \mathbf{C}(\epsilon)=\mathbf{C}(\epsilon)^{*} \mathbf{C}(\epsilon)=$ $\mathbf{W}^{H} \mathbf{W}=\mathbf{I}_{N}$, the resulting sequence after the DFT process is:

$$
\mathbf{s}_{l[\hat{\epsilon}=\epsilon]}=\mathbf{H}_{l} \mathbf{d}_{l} .
$$

In the case of $\mathrm{CM}$ signaling, i.e. $\left|d_{l}^{k}\right|=1$ and trying to focus on the amplitudes, the elements of the vector $\mathbf{s}_{l}$ will be expressed as:

$$
\left|s_{l}^{k}\right|=\left|H_{l}^{k}\right|, \quad k=0,1, \ldots, N-1
$$

If we assume that the channel response is slowly time-varying and therefore $\left|H_{l}^{k}\right|$ and $\left|H_{l+1}^{k}\right|$ are almost equal, i.e.

$$
\left|s_{l-1}^{k}\right|_{[\hat{\epsilon}=\epsilon]} \approx\left|s_{l}^{k}\right|_{[\hat{\epsilon}=\epsilon]},
$$

then, to estimate the value of $\epsilon$, we initially propose the following cost function:

$$
J_{i}(\tilde{\epsilon})=\sum_{l=1}^{M} \sum_{k=0}^{N-1}\left(\left|s_{l}^{k}\right|-\left|s_{l-1}^{k}\right|\right)^{2} .
$$

$\mathrm{CFO}$ is assumed to remain constant over $M$ contiguous OFDM symbols and $J_{i}(\tilde{\epsilon})$ should be minimized with respect to parameter $\tilde{\epsilon}$, which is the trial value of $\epsilon$. Thereby:

$$
\hat{\epsilon}=\arg \min _{\tilde{\epsilon} \in(-0.5,0.5)} J_{i}(\tilde{\epsilon}) .
$$

However, the two terms $\sum_{k=0}^{N-1}\left|s_{l}^{k}\right|^{2}$ and $\sum_{k=0}^{N-1}\left|s_{l-1}^{k}\right|^{2}$ are independent of $\tilde{\epsilon}$ and $\epsilon$ (see Appendix $\mathrm{A}$ for the proof). Consequently, minimizing (10) is reduced to maximizing the following final cost function with respect to $\tilde{\epsilon}$ :

$$
J_{f}(\tilde{\epsilon})=\sum_{l=1}^{M} \sum_{k=0}^{N-1}\left|s_{l}^{k}\right|\left|s_{l-1}^{k}\right|,
$$

so that we can deduce the $\mathrm{CFO}$ estimate value $\hat{\epsilon}$.

It is worth noting that, if we assume the OFDM symbol energy $\left(\sum_{k=0}^{N-1}\left|s_{l}^{k}\right|^{2}\right)$ to be almost insensitive to the Doppler effect, maximizing (12) could be seen as the maximization of the signal-to-interference-and-noise ratio $(S I N R)$ as introduced in [14]. Indeed, to estimate the CFO, the authors have proposed maximizing a frequency-oriented $S I N R$ estimate. However, our proposed scheme is based on a new approach exploiting channel coherence in time, appropriately. The optimization operation of (12) might be realized using several techniques. As the use of the gradient descent is not possible because the function is not differentiable, exhaustive search on a discrete set of values can be employed. However, it should be 


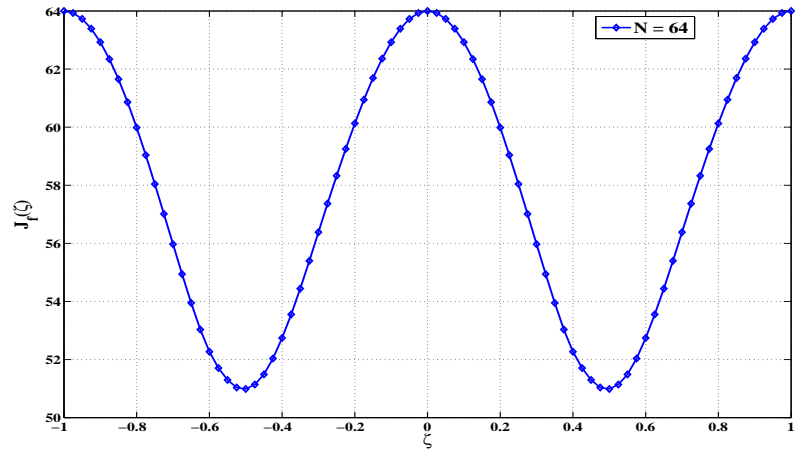

Fig. 1. $J_{f}$ versus $\zeta$ in case of $N=64$.

noted that the exhaustive search will require more computation time and complexity, thus making it impractical. Therefore, while seeking simplicity of implementation and using the basic assumption (9), we come up with a quasi-regular function, which is a good approximation of $J_{f}(\tilde{\epsilon})$, namely:

$$
J_{f}(\tilde{\epsilon}) \approx \alpha \cdot \cos [2 \pi(\epsilon-\tilde{\epsilon})]+\beta,
$$

where $\alpha$ and $\beta$ are constants, and above all independent of $\epsilon$ and $\tilde{\epsilon}$. They have positive real values and depend only on the frequency channel response and the sequence of information symbols. Through simulations, we always obtain a quasisinusoidal shape for $J_{f}(\zeta)$, where $\zeta=\epsilon-\tilde{\epsilon}$. The channel coefficients used are independent complex Gaussian random variables with zero mean and any power delay profile. In Fig. 1, we have considered a $T_{s}$-spaced 10-discrete-path fading channel, which has normally-distributed random taps with decaying power delay profile. Furthermore, in Appendix B, we prove that the function $J_{f}$ is 1-periodic and also even. Aiming to perform straightforward maximization operation with less computing time, we decided to make use of the curve-fitting process [11]. Similarly to the method description in [15], we first evaluate function (12) at three distinctive points: $\tilde{\epsilon}=a, b$ and $c$. Then, we compute the two intermediate paramameters $\gamma$ and $\delta$ defined as:

$$
\left\{\begin{array}{c}
\gamma:=\left[J_{f}(\tilde{\epsilon}=a)-J_{f}(\tilde{\epsilon}=c)\right] \cdot[\sin (2 \pi b)-\sin (2 \pi c)] \\
+\left[J_{f}(\tilde{\epsilon}=c)-J_{f}(\tilde{\epsilon}=b)\right] \cdot[\sin (2 \pi a)-\sin (2 \pi c)] \\
\delta:=\left[J_{f}(\tilde{\epsilon}=b)-J_{f}(\tilde{\epsilon}=c)\right] \cdot[\cos (2 \pi a)-\cos (2 \pi c)] \\
+\left[J_{f}(\tilde{\epsilon}=c)-J_{f}(\tilde{\epsilon}=a)\right] \cdot[\cos (2 \pi b)-\cos (2 \pi c)]
\end{array}\right.
$$

Finally, the CFO estimate is given by:

$$
\hat{\epsilon}=\frac{1}{2 \pi} \cdot \arg (\gamma+j \cdot \delta) .
$$

If we take $a=-1 / 4, b=1 / 4$ and $c=0$ in particular, (15) becomes simpler.

In comparison with [14], for implementation, we use the curve-fitting method, which is simple without any parameters to set, unlike the early-late-gate recovery loop implemented in [14].

The PDE (PDE-F and PDE-T) cost functions consist of terms where the signal amplitude is raised to the 4 th power.
Therefore, the impact of narrow-band noise on one subcarrier, or even more, would have a significant paralizing effect. Concerned subcarriers' terms will drastically reduce the contribution of the other subcarriers' terms in the sum. Thus, the value obtained by the estimation process will be considerably different from the real value. In contrast, (12) will experience much less degradation in case of narrow-band noise. This is due to the low power of all the elements of the sum. Furthermore, the gain control in digital communication systems is an inevitable process. Inappropriate increase or decrease in gain over one OFDM symbol period could often occur. In such a case, the ADE-T performance is not affected. Indeed, the desired parameter when maximizing $\sum_{k=0}^{N-1}\left|s_{l}^{k}\right|\left|s_{l-1}^{k}\right|$ (12) or $\sum_{k=0}^{N-1} \rho\left|s_{l}^{k}\right|\left|s_{l-1}^{k}\right|$ is exactly the same, where $\rho$ is a (non-zero) positive real number that corresponds to the gain variation on the $l$ th OFDM symbol and $k=0,1, \ldots, N-1$. Thus, the cost function used is gain variation independent unlike the function employed in [15].

\section{Simulation Results}

We consider a conventional quadrature phase-shift keying (QPSK) OFDM system having $N=64$ subcarriers with a cyclic prefix length of $N_{c p}=16$. Monte Carlo simulations were conducted on multipath Rayleigh fading channel using $10^{6}$ OFDM symbols on each simulation run. The channel varies every sampling period $T_{s}=\frac{T_{u}}{N}$ and Jakes' model [17] is used to generate the fading channel coefficients. $\epsilon$ is assumed to be uniformly distributed in the range $(-0.5,0.5)$ and keeps constant over two consecutive OFDM symbols $(M=1)$. It changes randomly from one pair of OFDM symbols to the next. The channel model used is identified by five path delays $\left[\begin{array}{lllll}0 & T_{s} & 2 . T_{s} & 6 . T_{s} & 11 . T_{s}\end{array}\right]$ with average power gains [0.34 0.28 0.23 0.11 0.04]. The normalized Doppler frequency $f_{d} T_{t}$ is set to different values, where $f_{d}$ designates the Doppler frequency. The underlying OFDM system operates at a central carrier frequency of $2.6 \mathrm{GHz}$ while the subcarriers' data rate is $16.68 \mathrm{~kb} / \mathrm{s}$. The regular mean squared error $(M S E)$ is used to assess the ADE-T performance with respect to that of PDE-F and PDE-T.

\section{A. General estimator performance analysis}

First, we perform the maximization process using the exhaustive search method with a precision of $10^{-3}$ by testing 1000 equally spaced $\tilde{\epsilon}$ candidates over the range $(-0.5,0.5)$. We are interested in this optimization method to get an idea about achievement limits as a benchmark for the three schemes in question. Fig. 2 shows the results from simulations conducted over a frequency-selective Rayleigh fading channel with $f_{d} T_{t}=0.030$ and 0.042 . These values correspond to vehicle speeds greater than $100 \mathrm{~km} / \mathrm{h}$. One can notice that the three estimators display very similar levels of performance. However, the exhaustive search method requires important memory resources and is too time-consuming to be practical for real-time wireless communication applications.

While being a good trade-off between performance and complexity, the curve-fitting method described in (14) and 


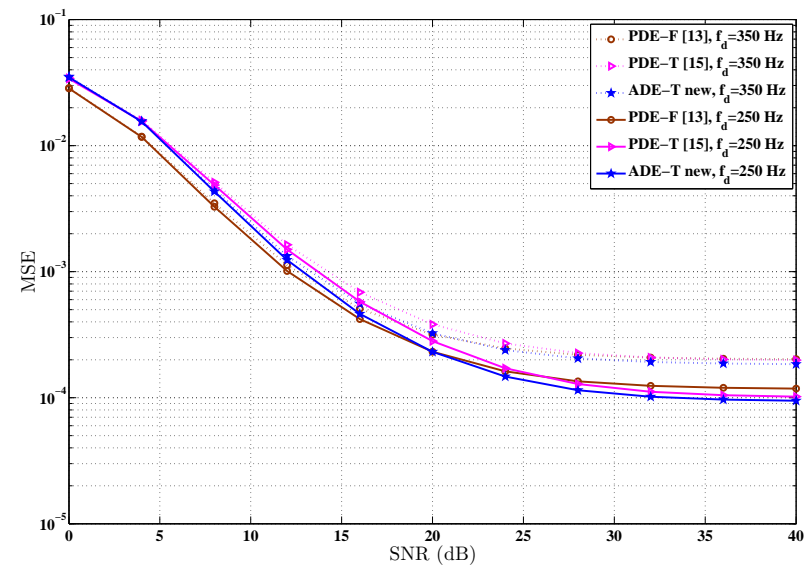

Fig. 2. $M S E$ versus the $S N R$ using the exhaustive search method over frequency-selective channel for $f_{d} T_{t}=0.030$ and 0.042 .

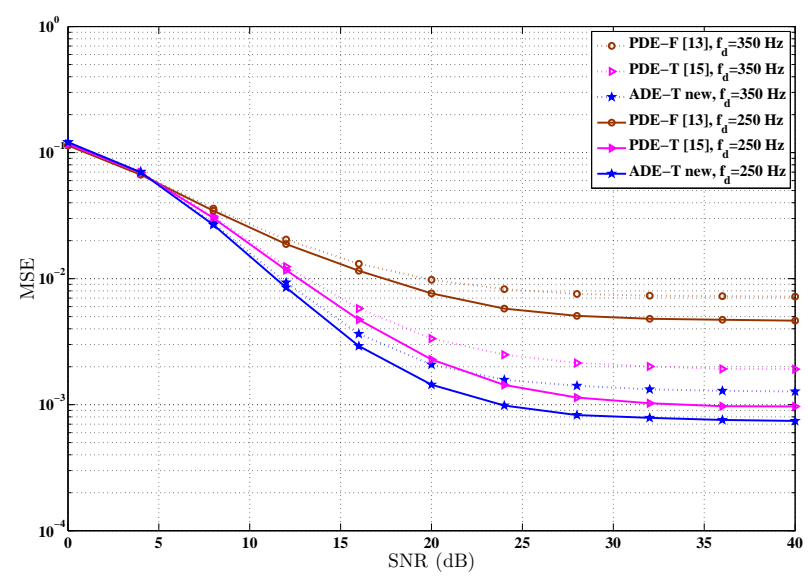

Fig. 3. MSE versus the SNR using the curve-fitting method over frequencyselective channel for $f_{d} T_{t}=0.030$ and 0.042 .

(15) is employed since the three functions targeted for optimization could be approximated by classic sine curves. Note that the exhaustive search method has involved at least 333 times more operations than curve-fitting. Fig. 3 shows the simulation results when the curve-fitting method is used for the three schemes. The first general observation to make is that, compared to the exhaustive search results, there is a performance loss of approximately one decade in terms of $M S E$. Nevertheless, ADE-T outperforms PDE-F and PDE-T for both values of $f_{d} T_{t}$ and over the entire range of $S N R$ s. The gain achieved with respect to PDE-T is less significant than that with respect to PDE-F. In the whole subsection coming after, the curve-fitting method will be used.

\section{B. Estimator performance exploration in particular scenarios of interest}

Let us now consider the potential contribution of the proposed method in another important context. Narrow-band noise is an interesting case from a practical perspective (cognitive radio, underwater acoustic communications,... etc).

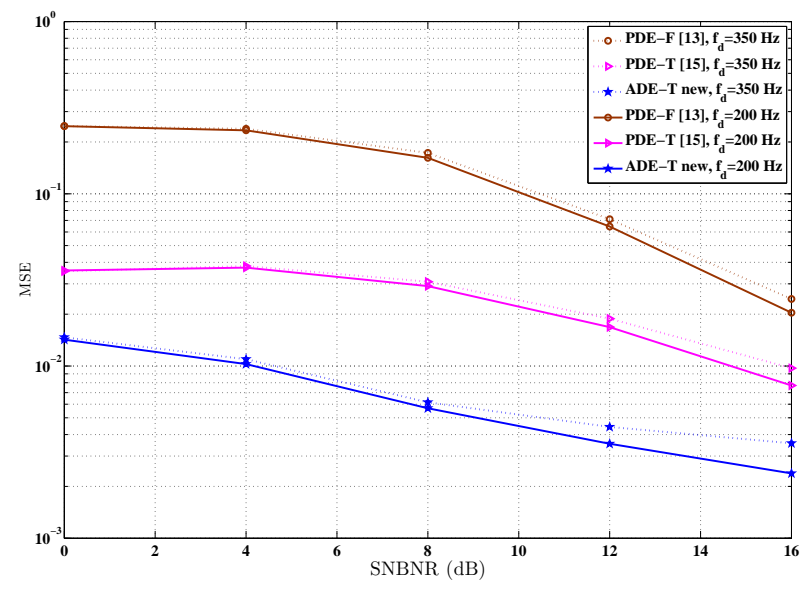

Fig. 4. MSE versus the signal to narrow-band noise ratio over frequencyselective channel for $f_{d} T_{t}=0.024$ and 0.042 .

The phenomenon is even more severe when interferers' operating frequencies are constantly changing. We evaluate the performance of the three schemes over a multipath fading channel introducing narrow-band noise. Results are given using $M S E$ versus the signal-to-narrow-band-noise ratio $(S N B N R)$, which is the mean power of emerging narrowband interferer signal at the receiver. Two values of the normalized Doppler frequency are used: $f_{d} T_{t}=0.024$ and 0.042. Performance levels obtained are depicted in Fig. 4 and we can see clearly that the ADE-T achievement is much greater than the other estimators.

The third scenario investigated implies operations of driving the automatic gain control (AGC) where the amplitude of the received signal varies over the duration of one OFDM symbol. As indicated in the previous section, one can identify the proposed estimator insentivity to gain changes, which is not the case for PDE-T. In order to pinpoint this issue, we have conducted simulations wherein we vary the gain in the range $(-3 \mathrm{~dB},+3 \mathrm{~dB})$. To compare the performance in such situations, the metric used once more is $M S E$ versus the gain variation occured in $\mathrm{dB}$. As demonstrated in Fig. 5 where $S N R=15 \mathrm{~dB}$, PDE-F and ADE-T are completely independent of the gain variation, unlike PDE-T which suffers from a penalizing sensitivity. Additionally, it is obvious that ADE-T outperforms PDE-F even if it is insensitive to gain fluctuations. For the same normalized Doppler frequencies $f_{d} T_{t}=0.030$ and 0.042 , and in case of $S N R=20 \mathrm{~dB}$, the $M S E$ performance presented in Fig. 6 confirm the estimators' behaviors.

\section{CONCLUSION}

In this paper, we presented a new method for blind CFO estimation in full loaded CM signaling based OFDM systems. It has been proven through numerical simulation results that the proposed low-complexity technique outperforms prominent existing estimators in various situations. In harsh time-varying propagation environments, modeled using a frequency-selective Rayleigh fading channel with Doppler 


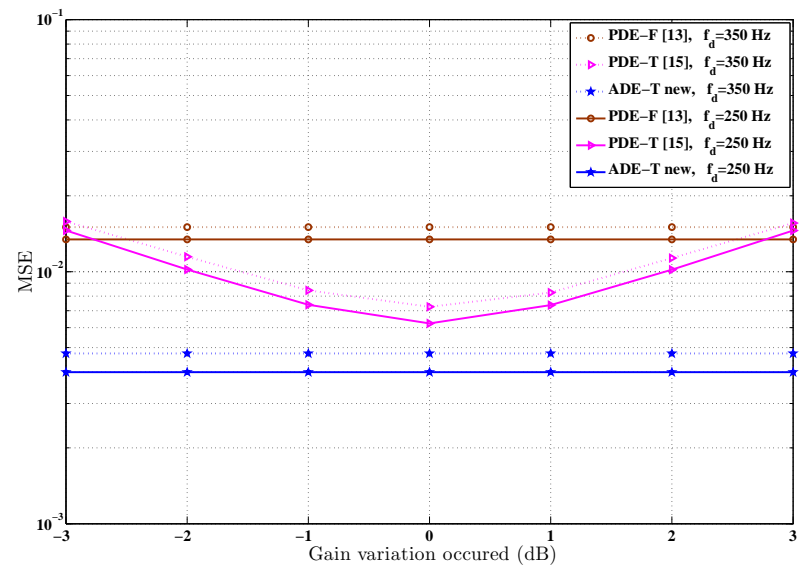

Fig. 5. MSE versus the gain variation occured on an OFDM symbol of two consecutive ones with $S N R=15 \mathrm{~dB}$ and for $f_{d} T_{t}=0.030$ and 0.042 .

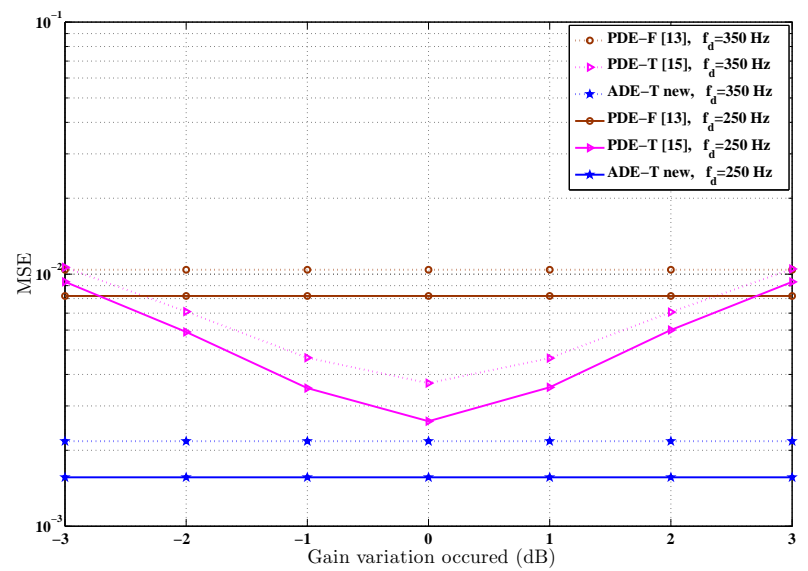

Fig. 6. MSE versus the gain variation occured on an OFDM symbol of two consecutive ones with $S N R=20 \mathrm{~dB}$ and for $f_{d} T_{t}=0.030$ and 0.042 .

shift, in a narrow-band noise context and in a situation involving fluctuating signal gain, the estimator introduced achieves very solid levels of performance. The efficiency carried out is due to the judicious manipulation of the received signal amplitudes to derive the appropriate cost function.

\section{APPENDIX A}

In the noise-free case, substituting (6) in (5) gives:

$$
s_{l}^{k}=\frac{e^{j 2 \pi \frac{\epsilon}{N}(l-1)\left(N+N_{c p}\right)}}{N} \sum_{n=0}^{N-1} \check{s}_{l}^{n} \sum_{m=0}^{N-1} e^{j 2 \pi \frac{m}{N}(n+\zeta-k)},
$$

where $\zeta=\epsilon-\tilde{\epsilon}$ and $\check{s}_{l}^{n}:=d_{l}^{n} H_{l}^{n}$. Since we are interested in the signal amplitude, we obtain:

$$
\begin{aligned}
& \sum_{k=0}^{N-1}\left|s_{l}^{k}\right|^{2}=\frac{1}{N^{2}} \sum_{n_{1}, n_{2}=0}^{N-1} \check{s}_{l}^{n_{1}} \check{s}_{l}^{n_{2} *} \\
& \quad \times \sum_{m_{1}, m_{2}=0}^{N-1} e^{j 2 \pi \frac{\zeta}{N} p} e^{j \frac{2 \pi}{N}\left(m_{1} n_{1}-m_{2} n_{2}\right)} \sum_{k=0}^{N-1} e^{-j 2 \pi \frac{k}{N} p},
\end{aligned}
$$

where $p=m_{1}-m_{2}$. Knowing that:

$$
\sum_{k=0}^{N-1} e^{-j 2 \pi \frac{k}{N} p}= \begin{cases}N, & \text { if } \quad p=0 \\ 0, & \text { otherwise }\end{cases}
$$

(16) becomes:

$$
\sum_{k=0}^{N-1}\left|s_{l}^{k}\right|^{2}=\frac{1}{N} \sum_{n_{1}, n_{2}=0}^{N-1} \check{s}_{l}^{n_{1}} \breve{s}_{l}^{n_{2}} * \sum_{m=0}^{N-1} e^{j \frac{2 \pi}{N} m q},
$$

where $q=n_{1}-n_{2}$ and $m_{1}=m_{2}=m$. Accordingly, $\sum_{k=0}^{N-1}\left|s_{l}^{k}\right|^{2}$ is completely independent of $\tilde{\epsilon}$ and $\epsilon$.

\section{APPENDIX B}

From (16) we have:

$$
N\left|s_{l}^{k}\right|=\left|\sum_{n=0}^{N-1} \check{s}_{l}^{n} \sum_{m=0}^{N-1} e^{j 2 \pi \frac{m}{N}(n+\zeta-k)}\right| .
$$

Therefore:

$$
\begin{aligned}
N^{2}\left|s_{l}^{k}\right|\left|s_{l-1}^{k *}\right|=\mid \sum_{n_{1}=0}^{N-1} \sum_{n_{2}=0}^{N-1} \check{s}_{l}^{n_{1}} \check{s}_{l-1}^{n_{2} *} \sum_{m_{1}=0}^{N-1}\left(e^{j \frac{2 \pi}{N}\left(n_{1}+\zeta-k\right)}\right)^{m_{1}} \\
\times \sum_{m_{2}=0}^{N-1}\left(e^{-j \frac{2 \pi}{N}\left(n_{2}+\zeta-k\right)}\right)^{m_{2}} \mid .
\end{aligned}
$$

Now, since:

$$
\begin{aligned}
\sum_{m_{1}=0}^{N-1}\left(e^{j \frac{2 \pi}{N}\left(n_{1}+\zeta-k\right)}\right)^{m_{1}} & =(-1)^{n_{1}-k} e^{\pi \zeta\left(1-\frac{1}{N}\right)} \\
& \times e^{j \pi\left(n_{1}-k\right)\left(1-\frac{1}{N}\right)} \frac{\sin (\pi \zeta)}{\sin \left[\frac{\pi}{N}\left(n_{1}+\zeta-k\right)\right]},
\end{aligned}
$$

and $e^{j \pi\left(n_{1}-n_{2}\right)}=e^{j \pi\left(n_{1}+n_{2}\right)}=(-1)^{n_{1}+n_{2}}=(-1)^{n_{1}-n_{2}}$ can be expressed as:

$$
\begin{aligned}
N^{2}\left|s_{l}^{k}\right|\left|s_{l-1}^{k *}\right|= & \mid \sum_{n_{1}=0}^{N-1} \sum_{n_{2}=0}^{N-1} \check{s}_{l}^{n_{1}} \check{s}_{l-1}^{n_{2} *} e^{j \pi \frac{n_{1}-n_{2}}{N}} \\
& \times \frac{\sin ^{2}(\pi \zeta)}{\sin \left[\frac{\pi}{N}\left(n_{1}+\zeta-k\right)\right] \sin \left[\frac{\pi}{N}\left(n_{2}+\zeta-k\right)\right]} \mid
\end{aligned}
$$

Hence, for $M=1$ (12) might be formulated as:

$$
\begin{aligned}
J_{f}(\zeta) & =\sum_{k=0}^{N-1}\left|s_{l}^{k}\right|\left|s_{l-1}^{k}\right|=\frac{1}{N^{2}} \sum_{k=0}^{N-1} \mid \sum_{n_{1}=0}^{N-1} \sum_{n_{2}=0}^{N-1} \check{s}_{l}^{n_{1}} \check{s}_{l-1}^{n_{2} *} \\
& \times e^{j \pi \frac{n_{1}-n_{2}}{N}} \frac{\sin ^{2}(\pi \zeta)}{\sin \left[\frac{\pi}{N}\left(n_{1}+\zeta-k\right)\right] \sin \left[\frac{\pi}{N}\left(n_{2}+\zeta-k\right)\right]} \mid .
\end{aligned}
$$

Now, let's show that $J_{f}(\zeta)$ is periodic with period 1. From (24) we have:

$$
\begin{aligned}
& J_{f}(\zeta+1)=\frac{1}{N^{2}} \sum_{k=0}^{N-1} \mid \sum_{n_{1}=0}^{N-1} \sum_{n_{2}=0}^{N-1} \check{s}_{l}^{n_{1}} \check{s}_{l-1}^{n_{2} *} e^{j \pi \frac{n_{1}-n_{2}}{N}} \\
& \times \frac{\sin ^{2}(\pi \zeta+\pi)}{\sin \left[\frac{\pi}{N}\left(n_{1}+\zeta-(k-1)\right)\right] \sin \left[\frac{\pi}{N}\left(n_{2}+\zeta-(k-1)\right)\right]} \mid
\end{aligned}
$$


which can be expressed otherwise when $k^{\prime}=k-1$ as:

$$
\begin{aligned}
& J_{f}(\zeta+1)=\frac{1}{N^{2}} \sum_{k^{\prime}=0}^{N-2} \mid \sum_{n_{1}=0}^{N-1} \sum_{n_{2}=0}^{N-1} \check{s}_{l}^{n_{1}} \check{s}_{l-1}^{n_{2} *} e^{j \pi \frac{n_{1}-n_{2}}{N}} \\
& \times \frac{\sin ^{2}(\pi \zeta)}{\sin \left[\frac{\pi}{N}\left(n_{1}+\zeta-k^{\prime}\right)\right] \sin \left[\frac{\pi}{N}\left(n_{2}+\zeta-k^{\prime}\right)\right]}\left|+\frac{1}{N^{2}}\right| \sum_{n_{1}=0}^{N-1} \sum_{n_{2}=0}^{N-1} \check{s}_{l}^{n_{1}} \\
& \quad \times \check{s}_{l-1}^{n_{2} *} e^{j \pi \frac{n_{1}-n_{2}}{N}} \frac{\sin ^{2}(\pi \zeta)}{\sin \left[\frac{\pi}{N}\left(n_{1}+\zeta+1\right)\right] \sin \left[\frac{\pi}{N}\left(n_{2}+\zeta+1\right)\right]} .
\end{aligned}
$$

Since:

$$
\begin{aligned}
& \sin \left[\frac{\pi}{N}\left(n_{1}+\zeta+1\right)\right] \sin \left[\frac{\pi}{N}\left(n_{2}+\zeta+1\right)\right] \\
& \quad=\sin \left[\frac{\pi}{N}\left(n_{1}+\zeta-(N-1)\right)\right] \sin \left[\frac{\pi}{N}\left(n_{2}+\zeta-(N-1)\right)\right]
\end{aligned}
$$

We obtain:

$$
\begin{aligned}
J_{f}(\zeta+1)= & \frac{1}{N^{2}} \sum_{k^{\prime}=0}^{N-1} \mid \sum_{n_{1}=0}^{N-1} \sum_{n_{2}=0}^{N-1} \check{s}_{l}^{n_{1}} \check{s}_{l-1}^{n_{2} *} e^{j \pi \frac{n_{1}-n_{2}}{N}} \\
& \times \frac{\sin ^{2}(\pi \zeta)}{\sin \left[\frac{\pi}{N}\left(n_{1}+\zeta-k^{\prime}\right)\right] \sin \left[\frac{\pi}{N}\left(n_{2}+\zeta-k^{\prime}\right)\right]} \mid,
\end{aligned}
$$

and therefore: $J_{f}(\zeta+1)=J_{f}(\zeta)$. Besides, let's prove that $J_{f}(\zeta)$ is even. Still from (24) we get:

$$
\begin{aligned}
J_{f}(-\zeta)= & \frac{1}{N^{2}} \sum_{k=0}^{N-1} \mid \sum_{n_{1}=0}^{N-1} \sum_{n_{2}=0}^{N-1} \check{s}_{l}^{n_{1}} \check{s}_{l-1}^{n_{2} *} e^{j \pi \frac{n_{1}-n_{2}}{N}} \\
& \times \frac{\sin ^{2}(\pi \zeta)}{\sin \left[\frac{\pi}{N}\left(-n_{1}+\zeta+k\right)\right] \sin \left[\frac{\pi}{N}\left(-n_{2}+\zeta+k\right)\right]}
\end{aligned}
$$

knowing that:

$$
\begin{aligned}
\sin \left[\frac { \pi } { N } \left(-n_{1}\right.\right. & +\zeta+k)] \sin \left[\frac{\pi}{N}\left(-n_{2}+\zeta+k\right)\right] \\
& =\sin \left[\frac{\pi}{N}\left(n_{1}^{\prime}+\zeta-k^{\prime}\right)\right] \sin \left[\frac{\pi}{N}\left(n_{2}^{\prime}+\zeta-k^{\prime}\right)\right],
\end{aligned}
$$

where $n_{1}^{\prime}=N-1-n_{1}, n_{2}^{\prime}=N-1-n_{2}$ and $k^{\prime}=N-1-k$. The term $e^{j \pi \frac{n_{1}-n_{2}}{N}}$ in (24) and (29) does not have any significant weight at all, since we can for instance take in the following equalities:

$$
\begin{aligned}
& \mid \sum_{n_{1}=0}^{N-1} \sum_{n_{2}=0}^{N-1} \check{s}_{l}^{n_{1}} \check{s}_{l-1}^{n_{2} *} e^{j \pi \frac{n_{1}-n_{2}}{N}} \\
& \quad \times \frac{\sin ^{2}(\pi \zeta)}{\sin \left[\frac{\pi}{N}\left(n_{1}+\zeta-k\right)\right] \sin \left[\frac{\pi}{N}\left(n_{2}+\zeta-k\right)\right]} \mid \\
& =\mid \sum_{n_{1}=0}^{N-1} \sum_{n_{2}=0}^{N-1} \check{s}_{l}^{n_{1}} \check{s}_{l-1}^{n_{2} *} e^{j \frac{\pi}{N}\left(n_{1}-n_{2}+N^{N+1}\right)} \\
& \quad \times \frac{\sin ^{2}(\pi \zeta)}{\sin \left[\frac{\pi}{N}\left(n_{1}+\zeta-k\right)\right] \sin \left[\frac{\pi}{N}\left(n_{2}+\zeta-k\right)\right]} \mid,
\end{aligned}
$$

where $n_{1}-n_{2}+N^{N+1} \approx N^{N+1}$ and $\left|e^{j \pi N^{N+1}}\right|=\left|e^{j \pi N^{N}}\right|=1$. We can conclude that: $J_{f}(-\zeta) \approx J_{f}(\zeta)$.

\section{REFERENCES}

[1] T.M. Schmidl and D.C. Cox, "Robust Frequency and Timing Synchronization for OFDM," IEEE Trans. Commun., vol. 45, no. 12, pp. 16131621, Dec. 1997.

[2] M. Morelli and U. Mengali, "An Improved Frequency Offset Estimator for OFDM Applications," IEEE Commun. Lett., vol. 3, no. 3, pp. 75-77, Mar. 1999.
[3] H. Minn, V.K. Bhargava, and K.B. Letaief, "A Robust Timing and Frequency Synchronization for OFDM Systems," IEEE Trans. Wireless Commun., vol. 2, no. 4, pp. 822-839, July 2003.

[4] G. Ren, Y. Chang, H. Zhang, and H. Zhang, "An Efficient Frequency Offset Estimation Method With a Large Range for Wireless OFDM Systems," IEEE Trans. Veh. Technol., vol. 56, no. 4, pp. 1892-1895, July 2007.

[5] S. Attallah, Y. Wu, and J.W.M. Bergmans, "Low complexity blind estimation of residual carrier offset in orthogonal frequency division multiplexing based wireless local area network systems," IET Commun., vol. 1, no. 4, pp. 604-611, Aug. 2007.

[6] B. Park, H. Cheon, E. Ko, C. Kang, and D. Hong, "A Blind OFDM Synchronization Algorithm Based on Cyclic Correlation," IEEE Signal Process. Lett., vol. 11, no. 2, pp. 83-85, Feb. 2004.

[7] M. Shi, Y. Bar-Ness, and W. Su, "Revisiting the Timing and Frequency Offset Estimation Based on Cyclostationarity with New Improved Method," IEEE Commun. Lett., vol. 13, no. 7, pp. 537-539, July 2009.

[8] M. Tanda, "Blind Symbol-timing and Frequency-offset Estimation in OFDM Systems with Real Data Symbols," IEEE Trans. Commun., vol. 52, no. 10 , pp. 1609-1612, Oct. 2004.

[9] Wen-Long Chin, "ML Estimation of Timing and Frequency Offsets Using Distinctive Correlation Characteristics of OFDM Signals Over Dispersive Fading Channels," IEEE Trans. Veh. Technol., vol. 60, no. 2, pp. 444-456, Feb. 2011.

[10] H. T. Hsieh and W. R. Wu, "Maximum Likelihood Timing and Carrier Frequency Offset Estimation for OFDM Systems With Periodic Preambles," IEEE Trans. Veh. Technol., vol. 58, no. 8, pp. 4224-4237, Oct. 2009.

[11] Y. Yao and G.B. Giannakis, "Blind carrier frequency offset estimation in SISO, MIMO, and multiuser OFDM systems," IEEE Trans. Commun., vol. 53, no. 1, pp. 173-183, Jan. 2005.

[12] X.N. Zeng and A. Ghrayeb, "A Blind Carrier Frequency Offset Estimation Scheme for OFDM Systems with Constant Modulus Signaling," IEEE Trans. Commun., vol. 56, no. 7, pp. 1032-1037, July 2008.

[13] X.N. Zeng and A. Ghrayeb, "CFO Estimation Schemes for Differential OFDM Systems," IEEE Trans. Wireless Commun., vol. 8, no. 1, pp. 124-129, Jan. 2009.

[14] W.L. Chin and S.G. Chen, "A Blind Synchronizer for OFDM Systems Based on SINR Maximization in Multipath Fading Channels," IEEE Trans. Veh. Technol., vol. 58, no. 2, pp. 625-635, Feb. 2009.

[15] A. Al-Dweik, A. Hazmi, S. Younis, B. Sharif, and C. Tsimenidis, "Carrier Frequency Offset Estimation for OFDM Systems Over Mobile Radio Channels," IEEE Trans. Veh. Technol., vol. 59, no. 2, pp. 974-979, Feb. 2010.

[16] S. Younis, A. Al-Dweik, C. Tsimenidis, B.S. Sharif, and A. Hazmi, "The Effect of Timing Errors on Frequency Offset Estimation in OFDM Systems," in Proc. 11th IEEE Int. Symp. Signal Process. and Inf. Technol., Bilbao, Spain, pp. 202-206, Dec. 2011.

[17] Theodore S. Rappaport, Wireless Communications: Principles And Practice, Prentice Hall, New Jersey, 2002. 